\title{
Editorial: Research methodology in library and information studies (LIS)
}

\section{8: Part Two}

Judith Broady-Preston

\section{Introduction}

In late 2017 LIR published part one of a special issue of the journal showcasing papers outlining and analysing contemporary research methodologies, models and methods of relevance to the information profession and the LIS discipline (Broady-Preston, 2017). The series of papers presented here constitutes the second and final part of this 2017/18 follow up to the original 2012 issue (Broady-Preston, 2012). As before, all papers are unique to the journal and have been through rigorous double blind peer review prior to acceptance. A member of the regular editorial team managed the second of the four papers co-authored by the guest editor through the editorial process. Thanks are due not only to Diane but again to all those colleagues who completed reviews.

The papers in this final part originate from research teams working in Australia, New Zealand, Thailand and the UK, ranging from work by authors with an established global reputation and others derived from doctoral theses successfully defended in the last two years.

\section{Papers}

All papers presented here identify and critically evaluate new or emerging models and frameworks available to researchers in the field. The four papers in this final part explore a range 
of predominantly qualitative research methods, the first of which from Howard analyses the Grounded Delphi Method (GDM), a relatively new methodology integrating aspects of Grounded Theory (GT) with the Delphi Method. Derived from a successfully defended doctoral thesis, this study represents only the third documented usage of the methodology at the time of submission (2017). Based in Australia, the research project explored the skills, knowledge, qualities and professional education needs of information professionals, to determine relevant educational requirements for those operating in an environment where cultural heritage boundaries were increasingly blurring.

Developed originally to overcome perceived deficiencies in both GT and Delphi, GDM improves the rigour of theory building in Delphi by following GT principles in the data analysis stages, employing the Straussian approach of open, axial and selective coding. Howard further suggests that the consensus aspects of Delphi form a useful addition to GT, assisting the researcher in deciding the relative importance of each category. In her opinion, GDM constitutes a viable alternative to standard GT or Delphi, and is particularly useful for "exploratory research in emerging research areas".

Continuing the learning theme, Limwichitr, Broady-Preston and Ellis describe research approaches for investigating the transformation of university libraries into learning organisations using a multi-case study design. The significance of such transformation for university libraries rests in its potential to reinforce their ability to remain relevant in a period of rapid and turbulent change in library and information services. An interpretivist lens, together with a qualitative case study approach was used in the study, based on an adaptation and extension of Garvin's Three 
Ms Framework, including identification and consideration of Critical Success Factors (CSFs). A unique model was developed as an outcome of the original study, allowing university libraries to investigate and transform their organisational culture, which is further transferable to a range of contexts and cultures.

An introduction to Indigenous research methodologies suitable for application to relevant areas of research inquiry in library and information sciences forms the subject of the third paper from Lilley, a New Zealand-based researcher. He introduces and defines indigenous research methods, also providing an assessment of their importance and application, including an example set in the New Zealand context.

Such a paper is timely; Lilley identifies an increased focus on indigenous activities at the international level, accompanied simultaneously by a growing awareness from countries with such populations that these groups have specific "resource and service needs". This increasing, emerging focus on indigenous information issues is yet to be matched or reflected in research studies in the discipline. Addressing the oft-expressed view that indigenous research needs to be conducted largely or solely by researchers of indigenous descent, Lilley highlights the scarcity of such researchers, recommending a partnership based approach between experienced nonindigenous researchers, community leaders and inexperienced indigenous students.

The final paper also represents a contribution to extending the range of theoretical frameworks and models available to researchers in the field. Johnson outlines a model for planning, developing and evaluating education for library, archive and information services, 
developed from Porter's Value Chain theory, and assessed in the context of a major study of development in Iraq. He concludes that the model not only serves as a useful framework for use when planning and evaluating education for service provision, but also may be adapted and used to assess contemporary professional practice and services, especially in the context of planning and evaluating change.

\section{Conclusion}

This final issue of the second volume of LIR addressing purely methodological issues in LIS research offers further evidence of the growing maturity of research in the field. The complex range of issues and problems present in contemporary professional practice require the use of an increasingly diverse range of frameworks and models to achieve an understanding of the forces and factors at play. It is a mark of maturity in the field that unique robust and rigorous extensions and alternatives to existing methodologies, addressing issues of relevance solely to the wider information profession, are developed and employed with confidence by a wide range of researchers.

\section{Reference}

Broady-Preston, J. (2012) Research methodology in library and information studies (LIS). Library and Information Research, 36, (112), 1-5.

\section{Acknowledgements}

Once again, my thanks to the editorial team of Library and Information Research for their exemplary patience in supporting a guest editor. 